University of South Carolina

Scholar Commons

10-1-1985

\title{
Temperature Dependence of the Normal-Metal Aharonov-Bohm Effect
}

\author{
S. Washburn \\ C. P. Umbach \\ R. B. Laibowitz \\ Richard A. Webb \\ University of South Carolina - Columbia, webbra@mailbox.sc.edu
}

Follow this and additional works at: https://scholarcommons.sc.edu/phys_facpub

Part of the Physics Commons

\section{Publication Info}

Published in Physical Review B, ed. Gene D. Sprouse, Volume 32, Issue 7, 1985, pages 4789-4792. Washburn, S. Umbach, C. P. Laibowitz, R. B., \& Webb, R. A. (1985). Temperature dependence of the normal-metal Aharonov-Bohm effect. Physical Review B, 32(7), 4789-4792. DOI: 10.1103/PhysRevB. 32.4789

(C) Physical Review B, 1985, American Physical Society accepted for inclusion in Faculty Publications by an authorized administrator of Scholar Commons. For more information, please contact digres@mailbox.sc.edu. 


\title{
Temperature dependence of the normal-metal Aharonov-Bohm effect
}

\author{
S. Washburn, C. P. Umbach, R. B. Laibowitz, and R. A. Webb \\ IBM Thomas J. Watson Research Center, P.O. Box 218, Yorktown Heights, New York 10598
}

(Received 2 July 1985)

\begin{abstract}
The amplitude of $h / e$ periodic oscillations in the magnetoresistance of very small normal-metal (Au) rings, as well as the harmonic $h / 2 e$, have been studied as a function of temperature. The amplitudes depend on the temperature $T$ roughly as $T^{-1 / 2}$, as expected from the averaging of conduction channels in the absence of inelastic scattering, but may not be entirely consistent with this model. At the lowest $T$, the size of the fluctuations in the conductance is about $\Delta G \sim e^{2} / h$, as predicted recently.
\end{abstract}

The observation of magnetoresistance oscillations periodic in the flux $h / e$ was reported recently for small loops of gold. $^{1}$ The observations confirmed a series of predictions ${ }^{2-4}$ that the fundamental period of the Aharonov-Bohm effect in normal metal loops is $h / e$. These predictions were maintained in spite of several experimental measurements ${ }^{5}$ of an $h / 2 e$ flux period (in long normal metal cylinders and arrays of rings), as well as several theoretical suggestions ${ }^{6,7}$ that $h / 2 e$ would be the fundamental period in any normal metal. The source of difference between the experiments and of the difference between the theoretical arguments is one and the same..$^{4,8}$ Namely, it is that in experimental samples or theoretical models that incorporate ensemble impurity averaging, the $h / 2 e$ period dominates. The study of the fundamental period $h / e$ requires models and devices that avoid this destructive averaging. In very small samples, there is the further complication of aperiodic fluctuations (AF) added to the magnetoresistance. ${ }^{9}$ The fluctuations result from the interference of the conduction electrons in the presence of magnetic flux piercing the wires which compose the device. ${ }^{10}$ The resistance changes aperiodically on the magnetic field scale of $\Delta H \sim(h / e) A$, where $A$ is the area of the metal projected normal to the field. Associated with the fluctuations is a random envelope function which modulates the amplitude of Aharonov-Bohm oscillations., ${ }^{11}$ Both the aperioidic and the periodic effects require that the electrons circle the loop coherently.

There are important characteristic lengths that can contribute to the physics of the $T$ dependence. One is the electron phase coherence length $L_{\phi}$, which is the distance that an electron travels before randomly changing its wavefunction phase. $L_{\phi}$ is, roughly speaking, the shorter of the inelastic diffusion length and the diffusion length between magnetic scattering events (which change the wave function by flipping the spin). The inelastic length is $\left(D \tau_{\text {in }}\right)^{1 / 2}$, where $D$ is the electron diffusion constant (assumed to be $T$ independent), and $\tau_{\text {in }}$ is the mean time between inelastic events. It is expected that $\tau_{\text {in }} \propto T^{-p}$ with $p \sim 1 .^{12}$ The magnetic length is probably independent of $T$. The ratios of these lengths to the sample length $L$ govern the size (or the presence) of the oscillations. There is also an energy "correlation length" $E_{c}$ which measures the energy $(T)$ scale over which the pattern of conductance fluctuations is not significantly changed. ${ }^{10}$ When $T$ is changed by an amount $\Delta T \leq E_{c}$ the conductance is not altered, since the distribution of conductance patterns has not changed. Only changes larger than this energy scale change the distribution of conduction states enough to change the pattern.

This Rapid Communication describes measurements of the temperature dependence of these new periodic oscillations $(h / e$ and $h / 2 e)$ and the aperiodic fluctuations in the magnetoresistance of single gold rings. We studied two rings made from a 38 -nm-thick polycrystalline gold film using fine line lithography. ${ }^{13}$ The samples were placed in the mixing chamber of a dilution refrigerator inside of an electrically shielded room. The devices were connected to the resistance meter through filters which isolated them from environmental noise. The resistance meter was a 4-probe, ac bridge comprising two "lock-ins" and a ratio voltmeter. The large ring was formed of wires $41 \mathrm{~nm}$ wide, had an inside diameter of $780 \mathrm{~nm}$, and a resistance $R_{0}=R(T$ $=4 \mathrm{~K}, H=0)=29 \Omega$; the small ring was formed of 37 $\mathrm{nm}$-wide wires, had an inside diameter of $285 \mathrm{~nm}$, and $R_{0}=76 \Omega$.

In Fig. 1(a), we display the magnetoresistance data for the large ring in the range $0<H<8 \mathrm{~T}$ (the limit of our solenoid). As seen in the insets, the oscillations persist without any sign of attenuation to the highest field reached.
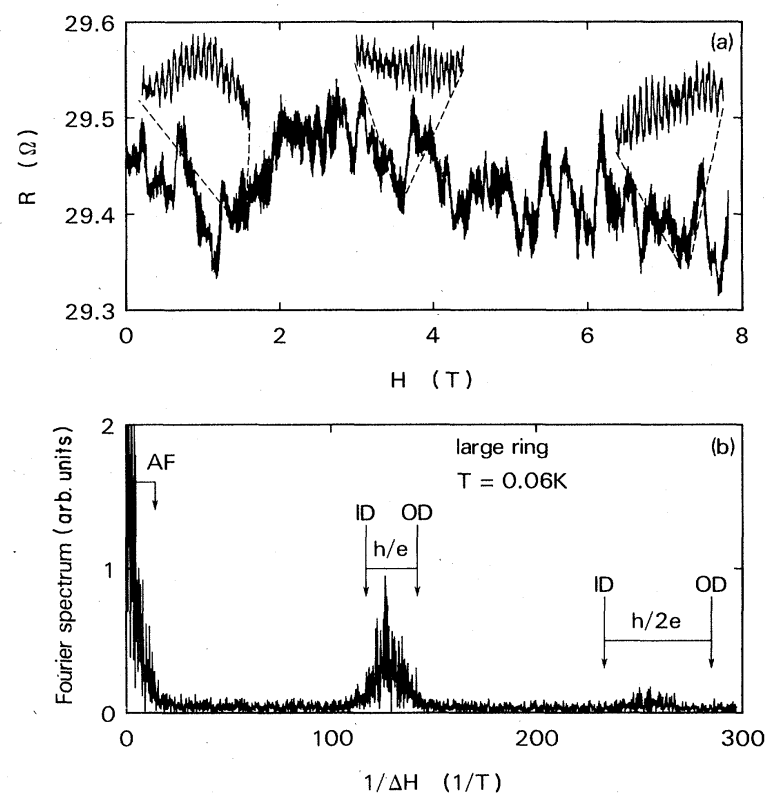

FIG. 1. (a) Magnetoresistance of the large ring. The insets are expanded views of the Aharonov-Bohm oscillations which illustrate the persistence of the oscillations to $8 \mathrm{~T}$. (b) Fourier spectrum containing peaks at $h / e$ and $h / 2 e$. The bars indicate the ranges of inverse field included in the sums for aperiodic fluctuations (AF), $h / e$, and $h / 2 e$ peaks. 
Figure 1(b) contains the Fourier spectrum of the data in Fig. 1(a). There are spectral peaks near $1 / \Delta H<14 \mathrm{~T}^{-1}$ corresponding to $\mathrm{AF}$, near $1 / \Delta H=130 \mathrm{~T}^{-1}$ corresponding to the $h / e$ Aharonov-Bohm oscillations, and near $1 / \Delta H=260 \mathrm{~T}^{-1}$ corresponding to the $h / 2 e$ period of oscillations. The magnetoresistance of the smaller ring is shown in Fig. 2 along with the Fourier spectrum. In this ring, there are spectral peaks near $1 / \Delta H=0, h / e, h / 2 e$, and $h / 3 e$. The presence of $h / 2 e$ oscillations in these high magnetic field data indicates that the coherent backscattering, ${ }^{14}$ which has been the source of $h / 2 e$ oscillations in previous experiments, ${ }^{5,6}$ is not responsible for these oscillations. For $L_{\phi} \geq L$ (which is believed to be necessary to observe the oscillations at all), the coherent backscattering process is destroyed ${ }^{14}$ within a few mT near $H=0$.

As noted previously, the spectrum is very complicated. ${ }^{1}$ The complexity is the signature of the random modulation function. ${ }^{11}$ This envelope function changes randomly once $T>E_{c}$. The distribution of conductance patterns is constant within $E_{c}$, but if the energy of the electrons is changed more than this scale then an uncorrelated set of states are conducting, which causes the background and the envelope to change shape. As it is the shape of the envelope that determines the detailed structure of the peaks in the Fourier spectrum, ${ }^{1,11}$ the individual spikes in the spectrum grow, move, or disappear randomly. This makes the extraction of useful information from the behavior of individual spikes impossible. To quantify the size of the spectral peaks for the fluctuations and the oscillations, we have summed the spectral weights over the regions indicated by the brackets. All of the data reported below were calculated by this method. The ranges encompassed by the brackets in Figs. 1(b) and 2(b) were given by the areas of the rings which were measured with an electron microscope to within an error $\leqslant 10 \%$. The spectral weight for the background fluctuations is summed over the range of frequencies allowed by the amount of wire between the voltage probes in the de-
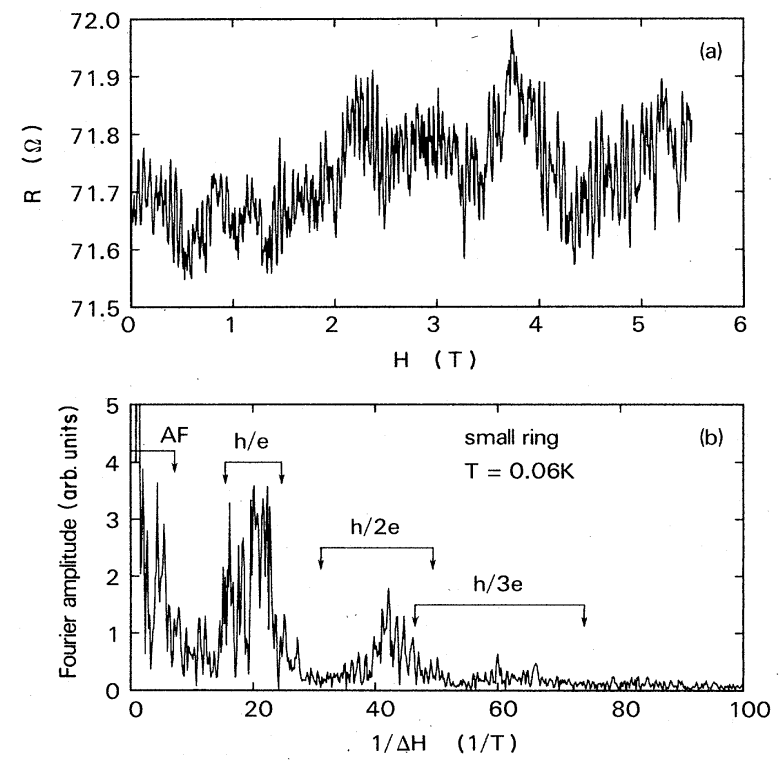

FIG. 2. Magnetoresistance (a) and Fourier spectrum (b) for the smaller ring. The spectral ranges for the various components of the magnetoresistance are shown by the arrows in (b) as in Fig. 1. vice. [This range is somewhat arbitrary. Numerical simulations of the magnetoresistance of similar wires have found that the fluctuations take place on the scale of $\Delta H z(2-4) h / e$ through the area covered by the wire. ${ }^{10}$ We have indicated $2 h / e$ on Figs. $1(b)$ and 2(b).] Similarly, the weights for the Aharonov-Bohm oscillations are summed over the range between the areas covered by the outside diameter and inside diameter of the ring.

The temperature dependence of the $h / e$ and $h / 2 e$ peaks from both rings is illustrated in Fig. 3, along with the dependence of the aperiodic fluctuations from the large ring. The immediate observation is that (with the possible exception of the $h / 2 e$ peak in the large ring) the data are consistent with the $T^{-1 / 2}$ dependence predicted for temperature averaging of the conductance states in the device. ${ }^{10}$ The AF peak, as reported previously, ${ }^{9}$ is consistent with $T^{-1 / 2}$. Both of the Aharonov-Bohm peaks in the small ring are $T$ dependent from $T=5 \mathrm{~K}$ down to $T \leqslant 0.1 \mathrm{~K}$, with the $h / e$ peak saturating at a slightly higher $T(0.08 \mathrm{~K})$ than the $h / 2 e$ peak $(0.04 \mathrm{~K})$. Both peaks are consistent with $T^{-1 / 2}$. For the large ring, neither of the Aharonov-Bohm peaks is discernible from the noise above $T \simeq 1 \mathrm{~K}$. Below $T \simeq 0.8 \mathrm{~K}$, the $h / e$ peak follows $T^{-1 / 2}$ down to approximately $0.03 \mathrm{~K}$. The $h / 2 e$ peak remains essentially lost in the noise down to $\simeq 0.2 \mathrm{~K}$, and then rises weakly.

The data from both rings allow the following intriguing possibility. The $T$ dependence of the $h / 2 e$ oscillations might be a weaker power law in temperature, illustrated by the solid line in Fig. $3(\mathrm{a})$, which crosses the $T^{-1 / 2}$ law near $1 \mathrm{~K}$. The implication of this is that in a ring which supports the Aharonov-Bohm effect to higher temperatures, the $h / 2 e$ period might dominate. Since the $h / 2 e$ amplitude is not much larger than the noise at the point where the lines cross, further experiments will be necessary before a conclusion can be reached. There is some theoretical support for this possibility. The $h / 2 e$ process may be indepen-

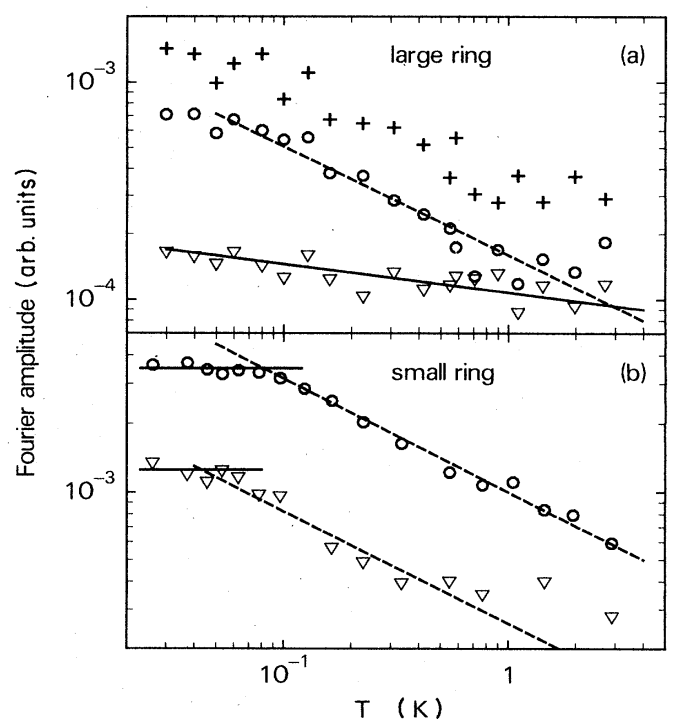

FIG. 3. Temperature dependence of the spectral peaks from the large (a) and small (b) rings. (,$+ \mathrm{AF} ; \mathrm{O}, h / e ; \nabla, h / 2 e$ ). These data result from sweeps of $-0.32 T<H<+0.32 \mathrm{~T}$ for the large ring (about $80 \mathrm{~h} / \mathrm{e}$ periods), and from sweeps between -1.1 and $+1.1 \mathrm{~T}$ for the small ring (about $45 \mathrm{~h} / e$ periods). The solid lines guide the eye, and the dashed lines illustrate the slope $T^{-1 / 2}$. 
$\operatorname{dent}^{8,15,16}$ of $E_{c}$.

Interpreting the data from the small ring in the framework of Ref. 10, we take the following view: that the saturation at relatively high (compared with the large ring) $T$ indicates that lowering $T$ further does not affect the distribution of states enough to change the conductance. (It is very unlikely that the saturation is an instrumental or heating problem, since both rings were measured under the same conditions.) This would imply that $E_{c}$ is of the order of $0.08 \mathrm{~K}$. This is certainly unexpected. A plausible estimate ${ }^{10}$ for $E_{c}$ is the spacing $\Delta E$ between the energy levels in the system which for metal samples the size of the small ring is $E_{c}=\Delta E \sim 10^{-4} \mathrm{~K}$. Imry ${ }^{16}$ has proposed the alternative energy scale first introduced by Thouless, ${ }^{17}$ namely, the sensitivity of the conductance to the boundary of the device $E_{c}=\left(h / \pi e^{2} R\right) \Delta E$ and this has received a firm theoretical footing. ${ }^{18}$ As the conductances of our samples are about a factor of 100-1000 greater than the fundamental conductance $e^{2} / h$, they make the latter estimate of the energy scale more attractive from the point of view of this experiment.

The tendency of the data to follow $T^{-1 / 2}$ is also evidence that $L_{\phi}$ is long compared to the diameters of the devices. If $L_{\phi}$ were much shorter than $L$, we would expect the model of Ref. 6 to be applicable. In rough terms, that model predicts that the oscillation amplitude is exponential in $L_{\phi}$, which would appear as an exponential in $T^{-p / 2}$. Although the data are not of sufficient quality to rule out an exponential completely, they are more consistent with the $T^{-1 / 2}$ dependence. Furthermore, the $L_{\phi}$ necessary to obtain the correct amplitude of oscillation from Ref. 6 is already larger than $L$; i.e., the samples appear to be in the regime studied in Refs. 4 and 10. The energy averaging theory cannot explain the difference between the saturation temperatures of the $h / e$ and the $h / 2 e$ peaks from the smaller ring. According to that formulation, once $T$ is smaller than $E_{c}$, the conductance is temperature independent. It should be noted that the energy averaging model does not predict a smooth $T^{-1 / 2}$ when $T \simeq E_{c}$, and the difference between the saturation temperatures may result from statistical fluctuations among the relatively small number of conduction patterns.

Assuming that $\Delta G \propto L_{\phi} \propto T^{-1 / 2}$ [following one-dimensional (1D) localization ${ }^{12}$ ] would, however, explain the difference between the two saturation temperatures very well. If $L_{\phi}$ is equal to the circumference of the loop at $T=0.1 \mathrm{~K}$, then it would be equal to $2 L_{\phi}$ at $T \simeq 0.03 \mathrm{~K}$ for $p=\frac{1}{2}$. This speculation is nearly in accordance with Fig. 3(b). The localization theory, however, would also predict that $R(T, H=0) \propto T^{-1 / 2}$. The prediction is violated by the data; in fact, the resistance of this ring increases with increasing temperature from $71.7 \Omega$ at $0.06 \mathrm{~K}$ to $76 \Omega$ at $T=4 \mathrm{~K}$.

At $T=0$, in any samples where $L_{\phi}>L$ and $T<E_{c}$, the $\mathrm{AF}$ in the magnetoconductance should have an amplitude ${ }^{18}$ of the order of the fundamental conductance $e^{2} / h$. This prediction agrees very well with the data reported here. From Fig. 1(a), the root-mean-square deviation from the average conductance is $\Delta G \simeq 1.1 e^{2} / h$ for the larger ring and, from Fig. $2(\mathrm{a}),=0.4 e^{2} / h$ for the smaller ring. (Analysis of a ring reported upon earlier ${ }^{9}$ yields $\Delta G \simeq 1.2 e^{2} / h$, also in agreement with the theory.) The amplitude of the fundamental Aharonov-Bohm oscillations also seems to be consistent with the theory. Filtering the magnetoresistance data to exclude everything but the $h / e$ oscillations, we obtain $\Delta G(h / e) \simeq 0.2 e^{2} / h$ from Figs. 1 (a) and 2 (a). It is not clear, however, that the theory ${ }^{18}$ applies to the Aharonov-Bohm oscillations.

To summarize, we have described the first measurements of the temperature dependence of the amplitude of the fundamental Aharonov-Bohm oscillations in a normal metal, and we have reported the dependence of the harmonic $h / 2 e$ and the aperiodic fluctuations. From the analysis of the temperature dependence we have concluded that the phasecoherence length $L_{\phi}$ of the electrons is larger than the device size $L$ for $T<0.1 \mathrm{~K}$, and may be larger than $L$ at much higher $T$. Considered in detail, the temperature dependence of the oscillations does not fit either the energy averaging theory or the theory of localization. At low temperature, the conductance fluctuations are of the order of the fundamental conductance $e^{2} / h$.

Note added in proof. Since submission of this manuscript, the theory has advanced considerably. Fukuyama, Lee, and Stone, ${ }^{19}$ using temperature-dependent Green functions, have predicted that the amplitude of the fluctuations in our devices should shrink with increasing temperature as $T^{-3 / 4}$.

The authors are grateful to D. Stone for several discussions of the energy averaging theory, and to P. A. Lee and D. Stone for explaining the new theory of conductance fluctuations prior to its publication. We also thank M. Büttiker, $Y$. Imry, and R. Landauer for numerous insightful comments on the data, and on the physics of the AharonovBohm effect in metals.
${ }^{1}$ R. A. Webb, S. Washburn, C. P. Umbach, and R. B. Laibowitz, Phys. Rev. Lett. 54, 2696 (1985).

${ }^{2}$ Y. Aharonov and D. Bohm, Phys. Rev. 115, 485 (1959).

${ }^{3}$ M. Büttiker, Y. Imry, and R. Landauer, Phys. Lett. 96A, 365 (1983); Y. Gefen, Y. Imry, and M. Ya. Azbel, Surf. Sci. 142, 203 (1984); Phys. Rev. Lett. 52, 129 (1984); M. Büttiker, Y. Imry, and M. Ya. Azbel, Phys. Rev. A 30, 1982 (1984).

${ }^{4}$ M. Büttiker, Y. Imry, R. Landauer, and S. Pinhas, Phys. Rev. B 31, 6207 (1985).

${ }^{5}$ D. Yu. Sharvin and Yu. V. Sharvin, Pis'ma Zh. Eksp. Teor. Fiz. 34, 285 (1981) [JETP Lett. 47, 272 (1981)]; Yu. V. Sharvin, Physica B 126, 288 (1984), and references cited therein.

6B. L. Al'tshuler, A. G. Aronov, and B. Z. Spivak, Pis'ma Zh. Eksp. Teor. Fiz. 3, 101 (1981) [JETP Lett. 33, 94 (1981)].
7J. P. Carini, K. A. Muttalib, and S. R. Nagel, Phys. Rev. Lett. 53, 102 (1984); D. A. Browne, J. P. Carini, K. A. Muttalib, and S. R. Nagel, Phys. Rev. B 30, 6798 (1984).

${ }^{8}$ Y. Gefen (unpublished); M. Murat, Y. Gefen, and Y. Imry (unpublished).

${ }^{9}$ R. A. Webb, S. Washburn, C. P. Umbach, and R. B. Laibowitz, in Localization, Interaction, and Transport Phenomena in Impure Metals, edited by G. Bergmann, Y. Bruynseraede, and B. Kramer (Springer-Verlag, Heidelberg, 1985); C. P. Umbach, S. Washburn, R. B. Laibowitz, and R. A. Webb, Phys. Rev. B 30, 4048 (1984).

${ }^{10}$ A. D. Stone, Phys. Rev. Lett. 54, 2692 (1985).

${ }^{11}$ A. D. Stone (unpublished); R. Landauer (unpublished).

12P. Santhanam, S. Wind, and D. E. Prober, Phys. Rev. Lett. 53, 


\section{RAPID COMMUNICATIONS}

1179 (1984), and references cited therein

${ }^{13}$ R. B. Laibowitz and A. N. Broers, in Treatise on Materials Science, edited by K. N. Tu and R. Rosenberg (Academic, New York, 1982), Vol. 24, p. 285.

${ }^{14}$ A. I. Larkin and D. E. Khmel'nitskii, Usp. Fiz. Nauk 136, 536 (1982) [Sov. Phys. Usp. 25, 185 (1982)]; G. Bergmann, Phys.
Rep. 107, 1 (1984).

${ }^{15} \mathrm{Y}$. Imry and N. Shiren (unpublished).

${ }^{16}$ Y. Imry (unpublished).

${ }^{17}$ D. J. Thouless, Phys. Rev. Lett. 39, 1167 (1977).

18P. A. Lee and A. D. Stone (unpublished).

${ }^{19}$ H. Fukuyama, P. A. Lee, and A. D. Stone (unpublished). 